\title{
Retrotransposons as a Genomic Risk Factor in Psychiatric Diseases
}

Yegin $\mathbf{Z}^{1^{*}}$, Avsar $\mathbf{C}^{2}$ and Sarisoy $\mathbf{G}^{3}$

${ }^{1}$ Medical Laboratory Techniques Program, Vocational School of Health Services, Sinop University, Sinop, Turkey

${ }^{2}$ Department of Biology, Faculty of Science and Arts, Sinop University, Sinop, Turkey

${ }^{3}$ Department of Psychiatry, Faculty of Medicine, Ondokuz Mayıs University, Samsun, Turkey

*Corresponding author: Yegin Z, Medical Laboratory Techniques Program, Vocational School of Health Services, Sinop University, Sinop, Turkey, E-mail: zyegin@sinop.edu.tr

Received date: October 03, 2018; Accepted date: October 20, 2018; Published date: October 27, 2018

Copyright: (c) 2018 Yegin Z, et al. This is an open-access article distributed under the terms of the Creative Commons Attribution License, which permits unrestricted use, distribution and reproduction in any medium, provided the original author and source are credited.

\section{Commentary}

In our DNA, there is a very small fraction (about 1\%) that is considered to be protein-encoding gene exons. There are intronic and intergenic genome regions filled with mobile DNA residues between these coding sequences. These are known as transposable elements and their ability to replicate over time shaped most of the modern human genome. In our DNA, we carry hundreds of thousands of copies of these sequences [1]. These DNA elements are called retrotransposons because of their ability to copy and paste themselves by using an RNA intermediate to insert their new copy into the main DNA. These retrotransposons are found in all eukaryotic genomes [2]. These retrotransposons, which are capable of introducing themselves into a new genomic position, are DNA fragments that cause mutations and alter the genomic landscape in the new integration region. Retrotransposons are the main class of transposable elements in all eukaryotic genomes [3] and have generated about $40 \%$ of the human genome [4]. Depending on the presence of Long Terminal Repeats (LTR) in their structure, they are classified into LTR and non-LTR retrotransposons. The LTR retrotransposon group which represents approximately $8 \%$ of the human genome consists of ERVs (Endogenous Retroviruses or Human Endogenous Retroviruses [HERVs]) that point to retrovirus sequences inserted into the host germline $[3,5]$. Non-LTR retrotransposon group is further divided into autonomous elements according to the ability of the elements to act independently or non-autonomous elements according to the mechanisms encoded by another retrotransposon and $\sim 1 / 3$ of human genome consists of non-LTR retrotransposons. Long interspersed element 1 (LINE-1 or L1) is the only autonomous non-LTR retrotransposon in humans. In addition to its own mobilization in the cis, L1 may activate in trans non-autonomous Short Interspersed Element (SINE) retrotransposons. These include 7 SL-derived Alu elements, composite SVA (SINE-variable number successive-Alu) elements and cellular mRNAs, resulting in processed pseudogenes. In studies conducted on these regions, copies of L1 were reported to be greater than $6 \mathrm{~kb}$, Alu copies were approximately $300 \mathrm{bp}$ and SVA copies were approximately $2 \mathrm{~kb}$ in size [3,6-8]. Considering the " $R N A$ world" representing the origin of life and the state of transformation into DNA by reverse transcription, it can be said that the mobile elements are participated much earlier in genome formation [9]. In this respect, it is reported that LTR retrotransposons, retroviruses HIV (Human Immunodeficiency Virus) and MLV (Murine Leukemia Virus) are quite similar in structure. The LTR retrotransposons and retroviruses provide enzymatic activities to make cDNA from RNA and place it in the genome, and include gag and pol genes that perform other tasks. It has been reported that non-LTR retrotransposons have a long history of 500 to 600 million years and they contain a RT similar to the RT of the moving group II introns that occur in the mitochondrial and chloroplast genomes of fungi and plants and some bacterial genomes [9-11]. For example, the genome of Saccharomyces cerevisiae contains five types of LTR retrotransposon elements, known as Ty1-5, which produces intracellular Virus-Like Particles (VLPs) [12]. Non-LTR retrotransposons Alu and SVA elements have increased in the last 80 million years of primate evolution and now account for about one third of the human genome together with L1. It is known that these elements affect the human genome in many different ways through addition mutations, genomic instability, changes in gene expression, and also genetic innovations. Since the sequences of human and other primate genomes are analyzed in more detail, the current contribution of non-LTR retrotransposons to the genomic change in the human line has begun to be demonstrated [13].

Retrotransposons can cause disease by different mechanisms, mainly by inactivating gene function through insertional mutagenesis or aberrant splicing. For instance, a LINE-1 insertion into an exon or an intronic region that is spliced may result in a frameshift mutation which will result in nonsense-mediated RNA decay. Another major mechanism by which LINE-1-mediated insertions result in disease may be through target-site deletions ranging from a few base pairs to megabase. An important hypothesis is that the insertions of LINE-1, Alu, and SVA elements may cause to the epigenetic changes at the site of integration which may result in reduced mRNA expression of the target gene [14]. Using high-throughput sequencing or other genomewide approaches, many previously unidentified L1 and Alu insertions and two HERV-K polymorphisms were identified. Unlike Single Nucleotide Polymorphisms (SNPs), retrotransposons may not just be linked to the causal variant but may indeed be the causal variant since they may affect gene expression [15]. De novo retrotransposition events are detectable in approximately 1 of 20 live births for Alu elements, 1 of 150 for LINE-1 and 1 of 1000 for SVA elements, and within the global human population there are an estimated 392 million private insertions unique to those harboring them [16]. Every human has on average $180 \mathrm{~L} 1,1283 \mathrm{Alu}$ and 56 SVA presence/absence insertion polymorphisms generating genetic variation [17]. Thus, the comprehensive analysis of these Retrotransposon Insertion Polymorphisms (RIPs) in different cohorts is quite advisable since the burden of RIPs within an individual may vary, and therefore the genes and pathways affected by their presence can differ [18].

The role of these viral remnants, called Human Endogenous Retroviruses (HERVs) in psychiatric disorders has been attracting attention in recent years. Approximately $50 \%$ of the human genome derives from unstable retro-elements and HERVs represent about $8 \%$ of chromosomal DNA [19]. Different types of HERVs have been described and classified as HERV-H, HERV-R, ERV-9 and HERV-K on the basis of their homologies. There are homologues of these viruses in 
non-human primates and each class seems to have a different evolutionary origin. Though the sequences are defective in a great majority of cases, expression has been detected in some cases; for instance HERV-E in placenta and various tumour cell lines, HERV-H, HERV-K and ERV-9 in teratocarcinoma cell lines and HERV-K in testicular tumours and at low levels in placenta and some normal tissues [20]. These ancient viruses infected the germline of the primate lineage multiple times over millions of years, and finally entered the human lineage. HERV-K represents the most recent HERV entrants in the human genome and besides the approximately 100 full-length HERV-K found in the genome, hundreds more HERV-K proviruses can be found dispersed throughout the centromeres of at least 15 human chromosomes [21,22]. Dr. Timothy Crow firstly proposed the role of retrotransposons in schizophrenia [23]. Since HERVs are polymorphic, they may contribute to genetic diversity among individuals and to susceptibility to common disorders. For instance, HERV-K element on chromosome 1 was associated with susceptibility to diabetes in individuals with schizophrenia [24,25]. The altered expression of HERV-W retroviral family was shown in schizophrenia, major depression, and bipolar disorder [26]. Moreover, the use of lithium or mood stabilizers did not have a significant effect on the expression of HERV-W GAG in bipolar disorder reflecting the minimal effect of medication on the expression of HERV-W, GAG [26]. The presence of HERV-W proteins in the serum of schizophrenic patients was analyzed with Enzyme-linked Immunosorbent Assay (ELISA) and HERV-W, GAG and ENV antigens were detected with significant levels in the sera of nearly $50 \%$ of patients [27]. HERV-W and ENV transcription levels were investigated in bipolar disorder and in schizophrenia as compared with healthy controls and an increased transcription rate was seen in Peripheral Blood Mononuclear Cells (PBMCs) of patients with bipolar disorder and schizophrenia; bipolar disorder group had a significantly higher expression than schizophrenia group. The corresponding DNA copy number was paradoxically lower in the genomes of patients with bipolar disorder or schizophrenia [28]. The possible role of HERVs was implicated in the pathophysiology of Attention Deficit Hyperactivity Disorder (ADHD). The expression levels of three HERV families (HERV-H, K and W) in PBMCs of patients with ADHD were investigated by quantitative RTPCR and levels of HERV-H were found significantly higher in patients with ADHD compared to healthy controls representing a new possible parameter of susceptibility to ADHD [29]. The reduction of HERV-H expression in a young drug-naive patient with ADHD after 6 months of Methylphenidate (MPH) treatment resulted with a significant improvement of symptoms reflecting the fact that transcriptional activation of certain retroviral elements may be associated with the disease [30]. L1 copy number in genomic DNA derived from postmortem brains of patients with major mental disorders were quantified and a significant increase of L1 content in the prefrontal cortex of patients with schizophrenia was reported [31]. In adult firstepisode schizophrenia patients, lower LINE-1 methylation was found to be associated with childhood adversities [32]. Bundo et al. [31], reported significant increases in L1 copy number in the prefrontal cortex of patients with schizophrenia. Thereafter, Doyle et al. [33], reported a significant increase in intragenic novel L1 insertions in dorsolateral prefrontal cortex of patients diagnosed with schizophrenia. Though there are only two common Alu insertion alleles were previously implicated in human phenotypes, now $44 \mathrm{Alu}$ insertion polymorphisms in strong linkage disequilibrium with the SNPs most associated with a disease phenotype were identified [34] and this issue may hold promise for psychiatric diseases as well.
In conclusion, the studies conducted up to now suggest the possible role of retrotransposons in the pathophysiology of psychiatric diseases. We strongly encourage further studies in this research field since possible signatures of susceptibility to psychiatric disorders can be evaluated with a new perspective (Since the burden of retrotransposon insertion polymorphisms may change within individuals and populations, it is logical to conduct the searches in different cohorts). This issue is also thrilling since it has the power of reflecting the effect of past in the future. In this framework, new studies will extend our scientific point of view on molecular evolution. Moreover, the involvement of retroviruses in disease pathogenesis may be exploited in future in terms of diagnosis and individualized treatment strategies.

\section{References}

1. Ardeljan D, Taylor MS, Ting DT, Burns KH (2017) The human long interspersed Element-1 Retrotransposon: An emerging biomarker of neoplasia. Clin Chem 63: 816-822.

2. Ballinger T, Ewing AD, Haussler D (2015) Retrotransposon mobilization in cancer genomes. arXiv preprint arXiv:1501.04268:1-16.

3. Bodea GO, McKelvey EG, Faulkner GJ (2018) Retrotransposon-induced mosaicism in the neural genome. Open Biol 8: 180074.

4. Goodier JL (2016) Restricting retrotransposons: A review. Mob DNA 7: 16.

5. Weiss RA (2016) Human endogenous retroviruses: Friend or foe?. APMIS 124(1-2): 4-10

6. Ullu E and Tschudi C (1984) Alu sequences are processed 7SL RNA genes. Nature 312: 171-172

7. Lander ES, Linton LM, Birren B, Nusbaum C, Zody MC, et al. (2001) Initial sequencing and analysis of the human genome. Nature 409: 860-921.

8. Ostertag EM, Goodier JL, Zhang Y, Kazazian Jr HH (2003) SVA elements are nonautonomous retrotransposons that cause disease in humans. Am J Hum Genet 73: 1444-1451.

9. Kazazian HH (2004) Mobile elements: Drivers of genome evolution. Science 303: 1626-1632.

10. Malik HS, Burke WD, Eickbush TH (1999) The age and evolution of nonLTR retrotransposable elements. Mol Biol Evol 16: 793-805.

11. Hou Y, Rajagopal J, Irwin PA, Voytas DF (2010) Retrotransposon vectors for gene delivery in plants. Mob DNA 1: 19.

12. Cheung S, Manhas S, Measday V (2018) Retrotransposon targeting to RNA polymerase III-transcribed genes. Mob DNA 9: 14 .

13. Cordaux R and Batzer MA (2009) The impact of retrotransposons on human genome evolution. Nat Rev Genet 10: 691.

14. Hancks DC, Kazazian HH Jr (2016) Roles for retrotransposon insertions in human disease. Mob DNA 7: 9.

15. Hancks DC, Kazazian HH Jr (2012) Active human retrotransposons: variation and disease. Curr Opin Genet Dev 22: 191-203.

16. Faulkner GJ (2011) Retrotransposons: Mobile and mutagenic from conception to death. FEBS Letters 585: 1589-1594.

17. Bennett EA, Coleman LE, Tsui C, Pittard WS, Devine SE (2004) Natural Genetic Variation Caused by Transposable Elements in Humans. Genetics 168: 933-951.

18. Savage AL, Schumann GG, Breen G, Bubb VJ, Al-Chalabi A, et al. (2018) Retrotransposons in the development and progression of amyotrophic lateral sclerosis. J Neurol Neurosurg Psychiatry pii: jnnp-2018-319210.

19. Lander ES, Linton LM, Birren B, Nusbaum C, Zody MC, et al. (2001) Initial sequencing and analysis of the human genome. Nature 409: 860-921.

20. Kim HS, Takenaka O, Crow TJ (1999) Isolation and phylogeny of endogenous retrovirus sequences belonging to the HERV-W family in primates. J Gen Virol 80: 2613-9.

21. Kim HS, Wadekar RV, Takenaka O, Hyun BH, Crow TJ (1999) Phylogenetic analysis of HERV-K LTR-like elements in primates: 
Citation: Yegin Z, Avsar C, Sarisoy G (2018) Retrotransposons as a Genomic Risk Factor in Psychiatric Diseases. J Clin Med Genomics 6: 150. doi:10.4172/2472-128X.1000150

Page 3 of 3

Presence in some new world monkeys and evidence of recent paralle evolution in these species and in homo sapiens. Arch Virol 144: 2035-40.

22. Zahn J, Kaplan MH, Fischer S, Dai M, Meng F, et al. (2015) Expansion of a novel endogenous retrovirus throughout the pericentromeres of modern humans. Genome Biol 16:74.

23. Crow TJ (1984) A re-evaluation of the viral hypothesis: Is psychosis the result of retroviral integration at a site close to the cerebral dominance gene?. Br J Psychiatry 145: 243-53.

24. Dickerson F, Rubalcaba E, Viscidi R, Yang S, Stallings C, et al. (2008) Polymorphisms in human endogenous retrovirus K-18 and risk of type 2 diabetes in individuals with schizophrenia. Schizophr Res 104: 121-6.

25. Torrey EF and Yolken RH (2008) The Past Is the Future. Biol Psychiatry 64: 1017-1018.

26. Weis S, Llenos IC, Sabunciyan S, Dulay JR, Isler L, et al. (2007) Reduced expression of human endogenous retrovirus (HERV)-W GAG protein in the cingulate gyrus and hippocampus in schizophrenia, bipolar disorder, and depression. J Neural Transm (Vienna) 114: 645-55.

27. Perron H, Mekaoui L, Bernard C, Veas F, Stefas I, et al. (2008) Endogenous Retrovirus Type W GAG and Envelope Protein Antigenemia in Serum of Schizophrenic Patients. Biol Psychiatry 64: 1019-1023.

28. Perron H, Hamdani N, Faucard R, Lajnef M, Jamain S, et al. (2012) Molecular characteristics of Human Endogenous Retrovirus type-W in schizophrenia and bipolar disorder. Transl Psychiatry 2: e201.
29. Balestrieri E, Pitzianti M, Matteucci C, D'Agati E, Sorrentino R, et al. (2014) Human endogenous retroviruses and ADHD. World J Biol Psychiatry 15: 499-504.

30. D'Agati E, Pitzianti M, Balestrieri E, Matteucci C, Sinibaldi Vallebona P, et al. (2016). First evidence of HERV-H transcriptional activity reduction after methylphenidate treatment in a young boy with ADHD. New Microbiol 39: 237-239.

31. Bundo M, Toyoshima M, Okada Y, Akamatsu W, Ueda J, et al. (2014) Increased L1 retrotransposition in the neuronal genome in schizophrenia. Neuron 81: 306-13.

32. Misiak B, Szmida E, Karpiński P, Loska O, Sąsiadek MM, et al. (2015) Lower LINE-1 methylation in first-episode schizophrenia patients with the history of childhood trauma. Epigenomics 7: 1275-85.

33. Doyle GA, Crist RC, Karatas ET, Hammond MJ, Ewing AD, et al. (2017) Analysis of LINE-1 Elements in DNA from Postmortem Brains of Individuals with Schizophrenia. Neuropsychopharmacology 42: 2602-2611.

34. Payer LM, Steranka JP, Yang WR, Kryatova M, Medabalimi S, et al. (2017) Structural variants caused by Alu insertions are associated with risks for many human diseases. Proc Natl Acad Sci USA 114: E3984-E3992. 